\title{
Toints
}

$\sqrt{2}$

\section{Bipolar fresh osteochondral allograft of the shoulder}

\author{
SANDRO GIANNINI, ELISA SEBASTIANI, ALBA SHEHU, MATTEO BALDASSARRI \\ SUSANNA MARALDI, FRANCESCA VANNINI
}

$1^{\text {st }}$ Clinic of Orthopaedics and Traumatology, Rizzoli Orthopaedic Institute, Bologna, Italy

\begin{abstract}
Purpose: to describe an original technique and preliminary results of bipolar fresh osteochondral allograft implantation for the treatment of end-stage glenohumeral osteoarthritis.

Methods: three patients underwent bipolar fresh osteochondral allograft implantation to the shoulder. Clinical and radiographical evaluations were carried out periodically through to final follow-up.

Results: constant Score increased from $38.3 \pm 2.9$ preoperatively to $78.7 \pm 16.2$ at 12 months, $72.3 \pm 15.3$ at 24 months, and $59.3 \pm 22.0$ at 34 months. Arthritis and partial reabsorption of the implanted surfaces were evident radiographically.

Conclusions: the clinical results obtained in these patients seem to support the applicability of bipolar fresh osteochondral allograft implantation in the shoulder in subjects with severe post-traumatic arthritis and intact rotator cuff. The development of arthritis of the implanted surfaces, while not impacting the clinical result, is a cause of concern.

Level of evidence: level IV, therapeutic case series.
\end{abstract}

Key Words: allograft, arthritis, bipolar fresh osteochondral allograft, shoulder.

\section{Introduction}

Shoulder pain due to osteoarthritis of the glenohumeral joint is a therapeutic challenge for the surgeon (1, $2)$. In patients with an intact rotator cuff, the most

\footnotetext{
Corresponding Author:

Francesca Vannini MD, PhD

$1^{\text {st }}$ Clinic of Orthopaedics and Traumatology,

Rizzoli Orthopaedic Institute, Bologna, Italy

Phone: +39 051/6366669

E-mail: France_vannini@yahoo.it
}

widely used surgical options are total shoulder arthroplasty and hemiarthroplasty with or without biological glenoid resurfacing, even in patients younger than 55 years $(3,4)$.

Nevertheless, shoulder arthroplasty in young patients carries a high percentage of unsatisfactory results, even though recent reports have shown favorable outcomes in the short to medium term (5-7).

Bipolar fresh osteochondral allograft (BFOA) placement, first proposed for the ankle and knee joint, is a fascinating option for biological joint reconstruction (8-14). The rationale of BFOA is to provide viable cartilage that can survive transplantation, being supported by an intact subchondral bony structure, which is progressively integrated and replaced by host bone over time $(12,15,16)$.

To our knowledge, the literature contains no reports of bipolar total allograft procedures applied to the shoulder $(12,17)$.

The purpose of the present study was to describe the surgical technique for BFOA of the shoulder and to report the outcome of BFOA of the shoulder in three cases at more than 30 months' follow-up.

\section{Methods}

Three patients (two men and one woman), mean age $44.0 \pm 3.6$ years, were referred for evaluation of longstanding shoulder pain and mechanical symptoms at the authors' institute. The diagnosis of post-traumatic arthritis of the glenohumeral surfaces with intact rotator cuff was confirmed by MRI. Substitution of the bare articular surfaces with a fresh bipolar allograft was indicated as a surgical option. The proposed procedure was approved by the institute's ethics committee and informed consent to the procedure was obtained from the patients. 
The affected shoulder of each patient was examined in order to assess the level of pain and the functional impairment. Clinical evaluation before allograft reconstruction was performed and rated according to the Constant Score. Radiographic evaluation was performed with standard radiographs and a CT scan of the affected shoulder was also performed in order to digitally measure the size of the shoulder. The supero-inferior and antero-posterior diameters of the glenoid were taken into account, together with the diameter of the humeral head considered as a sphere, and used to match the donor size with the recipient site.

The candidates were put on a waiting list pending the availability of an appropriate-sized donor joint. The donors were identified through our institute's Bone Bank program for musculoskeletal tissue transplantation. Using a standard surgical procedure, in a sterile regimen, the entire shoulder joint was harvested "en bloc" from each donor. Once harvested, a mini-incision was performed in the capsule in order to visually check the status of the cartilage and to allow diffusion of the storage medium. Following a CT scan, the allograft was placed in a sterile container with L-glutamine, NaHCO3 and antibiotic solution and stored at $4^{\circ} \mathrm{C}$. A MRI was finally performed on the harvested shoulder in order to verify articular cartilage integrity, while the adequacy of the shoulder size for the patient was verified through a CT scan. Allograft implantation was performed $14.0 \pm 1.0$ days after harvesting. The surgical session consisted of two steps: graft preparation and graft implantation.

Step 1. On a separate surgical table, all soft tissues were carefully removed from the harvested shoulder, taking care not to damage the cartilage. Then the articular surfaces of the glenoid and the humeral head were carefully cut with a standard pneumatic saw, keeping the whole articular surface and about 10-12 $\mathrm{mm}$ of subchondral bone intact. The prepared articular surfaces were then temporarily placed in a container of saline solution.

Step 2. The patient was placed in the beach-chair position under general anesthesia. A standard deltoid-pectoral approach was performed. With a blunt dissection the cephalic vein was identified and dislocated laterally. The subscapularis muscle was then identified and detached from its insertion. The capsule was opened and the humeral head was dislocated anteriorly. Under fluoroscopic control two Kirschner wires were placed in the humeral neck (perpendicular to the axis of the neck) and another two Kirschner wires were placed in the base of the glenoid, parallel to the articular surface, in order to define the plane for the bone cut (Fig. 1). The cut was then made using a standard pneumatic saw. The articular surfaces of the humeral head and the glenoid were removed taking care to remove the whole articular surfaces and $1 \mathrm{~cm}$ of subchondral bone (Fig. 2). Finally, the articular surfaces were replaced with the previously prepared allograft components.

The newly implanted glenoid was fixed with two small fragment screws, whereas the humeral head was fixed with two twist-off screws (Fig. 3). The joint was reduced and fluoroscopic control was performed in order to verify the correct placement of the graft; the shoulder range of motion (ROM) was also checked. Finally, the subscapularis muscle was reinserted, a drainage tube was placed and a routine suture was performed. Postoperative radiographs were taken.

Each patient underwent a light postoperative immunosuppressive therapy regimen: cyclosporin $3 \mathrm{mg} / \mathrm{kg}$ per day for six months and prednisone $10 \mathrm{mg}$ per day for the first month then $5 \mathrm{mg}$ per day for a further two months. Nine to 12 months after surgery the patients underwent a second operation for removal of the hardware and biopsy evaluation of the implanted allograft. Biopsy samples of cartilage, bone and synovial tissue were taken and immunological and immunohistochemical analysis of the specimens was carried out. The Constant Score at 12, 24 and 34 months after surgery was used to assess the clinical outcome.

\section{Results}

No intraoperative or postoperative complications occurred.

At final follow-up all the patients had a full and painless ROM with no additional complaints of resting pain or pain related to activities; the Constant Score increased from $38.3 \pm 2.9$ preoperatively to $78.7 \pm$ 16.2 at 12 months, $72.3 \pm 15.3$ at 24 months and 59.3 \pm 22.0 at 34 months. Two patients declared themselves to be satisfied with the treatment and the result. One patient was not satisfied with the treatment 


\section{oints}

S. Giannini et al.

$\sqrt{0}$

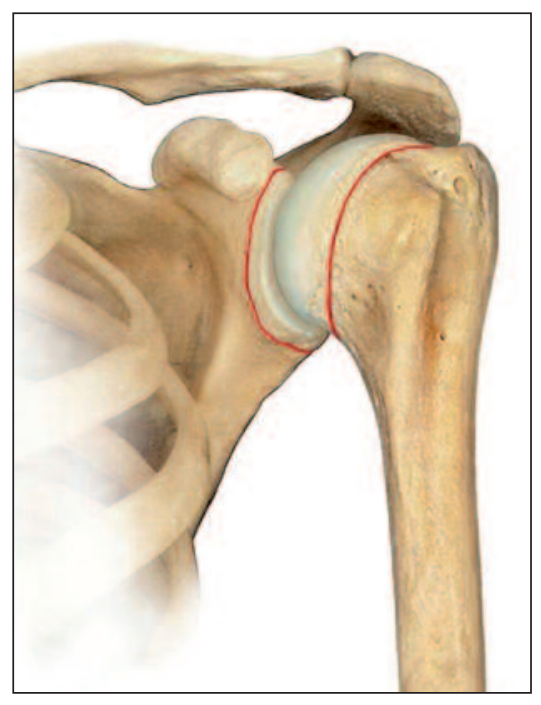

Fig. 1. Illustration showing the line of the cut on the recipient surfaces.

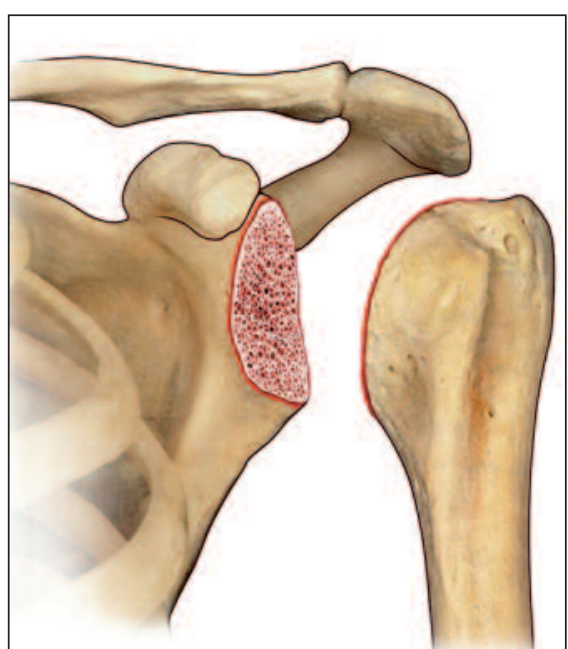

Fig. 2. The articular surfaces of the humeral head and the glenoid were removed taking care to remove the whole articular surfaces and $1 \mathrm{~cm}$ of subchondral bone.

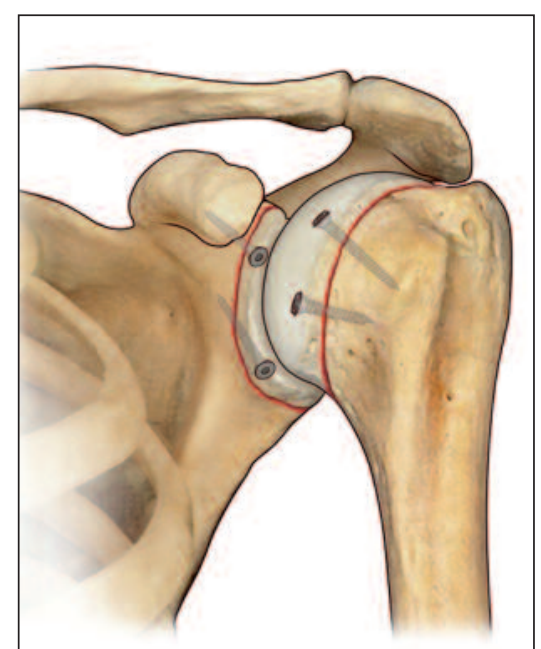

Fig. 3. Illustration showing the correct position of the allograft. The newly implanted glenoid was fixed with two small fragment screws, whereas the humeral head was fixed with two twist-off screws. because of instability and reduced strength, but to date has refused revision surgery.

All the allografts showed signs of integration on CT scan at four months of follow-up and were considered healed at six months on the basis of X-ray results. Follow-up MRI at six months showed a well-maintained cartilage layer in all cases. There was no intra-articular effusion, although subchondral bone edema was evident. No partial reabsorption of the implanted surfaces was evident at the final follow-up.

The degree of osteoarthritis was not found to impact on the clinical score at any follow-up, nor was it influenced by the immunosuppressive therapy.

Immunohistochemical analysis of the biopsy specimens revealed vital cartilage with a high proteoglycan content, normal cell distribution and type 2 collagen expression. Moreover, on the bony interface, the biopsy showed complete osseointegration between the graft and the recipient bone (Fig. 4). Finally, synovial biopsy analysis revealed a limited number of macrophages, without prominent perivascular inflammatory cell infiltrates or lymphoid aggregates.

\section{Discussion}

Bipolar fresh osteochondral allograft was found to be a viable option for the treatment of severe post-trau- matic arthritis of the shoulder in the presence of an intact rotator cuff. The technique allowed biological resurfacing and may represent a fascinating alternative to traditional prosthetic substitution.

It has been established that BFOA is applicable for treatment of the ankle joint, however, only partial or monopolar allografts have, to date, been reported in other joints $(11,14,17-20)$. Although the concept of allograft implantation is well established in the treatment of shoulder instability (21) and in revision shoulder arthroplasty (15), there are no reports of bipolar total fresh allografting.

The arthritic shoulder may represent an ideal indication for BFOA implantation since it is a non-weightbearing joint, but is subject to compressive and shear forces that can easily lead to mobilization of prosthetic components; the technique was previously presented in a single case report (16). The recipient showed complete integration of the allograft, which allowed him to achieve almost normal shoulder biomechanics and therefore an almost normal shoulder ROM (16).

These results were confirmed in the present case series, even though the number of patients considered is very small: shoulder ROM was restored and the patients were pain free. Unfortunately, one case developed laxity of the joint, which was the cause of the significant decrease in this patient's final score. 


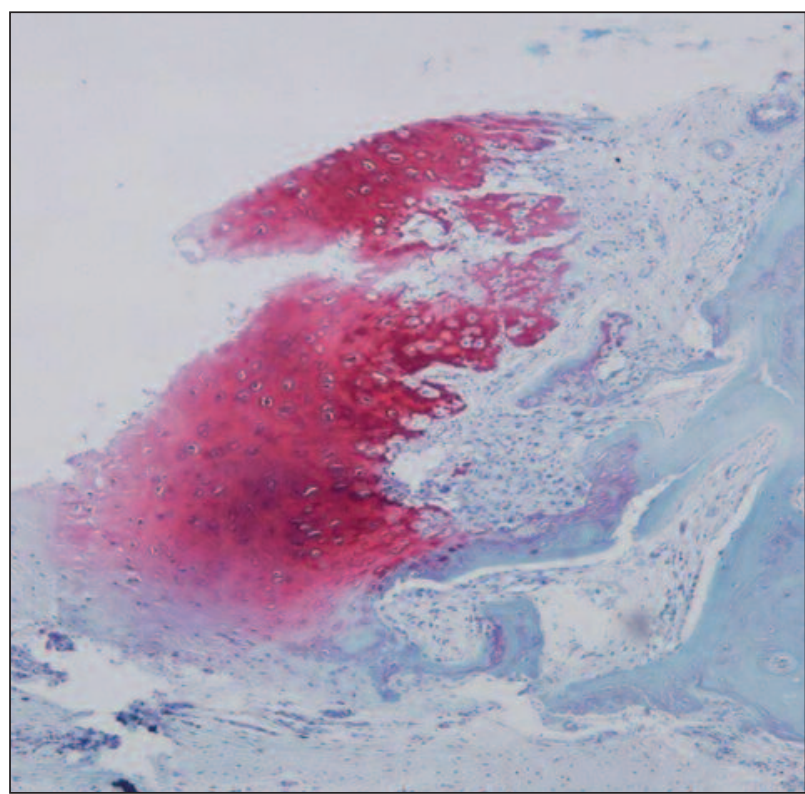

Fig. 4. Histological findings of the allograft. Biopsy specimen showed vital cartilage with a high proteoglycan content, normal cell distribution and complete osseointegration between the graft and the recipient bone. Safranin-O staining. Magnification 20x.

The immunosuppressive therapy was performed on the basis of the idea that the immune system plays a role in rejection of transplanted cartilage; the aim was to prevent the resulting damage from causing arthritis (22-24). The protocol we used was a light immunosuppressive therapy, already used in rheumatology, which was administered for six months. This protocol was easily tolerated by the patients and no adverse events were noted, however it was found to show poor protective efficacy against arthritis. Further studies are required in order to understand how to prevent arthritic changes in transplanted cartilage.

BFOA is a technically demanding surgical procedure, requiring a multidisciplinary approach and a bone bank facility. The significance of the present work lies in the fact that its results support the applicability of BFOA as an interesting surgical alternative to traditional arthroplasty in severe post-traumatic shoulder arthritis in young, active patients. The limited number of patients and the short follow-up are limitations of this analysis. Nevertheless, the fact that arthrodesis is poorly applicable for the shoulder, and shoulder replacement has limited indications in young patients, makes this possible alternative solution extremely appealing. However, the indications for its application are extremely limited because of the need for an intact rotator cuff.

In conclusion, the clinical results obtained in the cases described were extremely satisfactory in terms of pain resolution, ROM, overall function of the transplanted shoulder, and patient satisfaction and quality of life. In one patient, however, the development of shoulder laxity led to clinical impairment; to date, no revision surgery has been performed in this patient. The histological results also confirmed good survivorship of the chondrocytes after implantation and good integration of the graft. In all the patients, despite correct graft positioning and size, the radiographic results at final follow up were disappointing, even though they did not impact on the clinical result.

\section{References}

1. Sperling JW, Cofield RH, Rowland CM. Minimum fifteenyear follow up of Neerhemiarthroplasty and total shoulder arthroplasty in patients aged fifty years or younger. J Shoulder Elbow Surg 2004; 13:604-613.

2. Bailie DS, Llinas PJ, Ellenbecker TS. Cementless humeral resurfacing arthroplasty in active patients less than fifty-five years of age. J Bone Joint Surg Am 2008; 90:110-117.

3. Meehan R, McFarlin S, Bugbee W, et al. Fresh ankle osteochondral allograft transplantation for tibiotalar joint arthritis. Foot Ankle Int 2005; 26:793-802.

4. Weng PW, Shen HC, Lee HH, et al. Open reconstruction of large bony glenoid erosion with allogeneic bone graft for recurrent anterior shoulder dislocation. Am J Sports Med 2009; 37:1792-1797.

5. Langer F, Gross AE. Immunogenicity of allograft articular cartilage. J Bone Joint Surg Am 1974; 56:297-304.

6. Shasha N, Aubin PP, Cheah HK, et al. Long-term clinical experience with fresh osteochondral allografts for articular knee defects in high demand patients. Cell Tissue Bank 2002; 3:175-182.

7. Provencher MT, Leclere LE, Ghodadra N, et al. Postsurgical glenohumeral anchor arthropathy treated with a fresh distal tibia allograft to the glenoid and a fresh allograft to the humeral head. J Shoulder Elbow Surg 2010; 19:e6-e11.

8. Garrett JC. Treatment of osteochondral defects of the distal femur with fresh osteochondral allografts: a preliminary report. Arthroscopy 1986; 2:222-226.

9. Convery FR, Meyers MH, Akeson WH. Fresh osteochondral allografting of the femoral condyle. Clin Orthop Relat Res 1991; 273:139-145.

10. Chu CR, Convery FR, Akeson WH, et al. Articular cartilage transplantation. Clinical results in the knee. Clin Orthop Relat Res 1999; 360:159-168.

11. Bugbee WD, Convery FR. Osteochondral allograft transplantation. Clin Sports Med 1999; 18:67-75.

12. Hopkins AR, Hansen UN, Amis AA, et al. Wear in the prosthetic shoulder: association with design parameters. J Biomech Eng 2007; 129:223-230.

13. Levy JC, Virani NA, Frankle MA, et al. Young patients with 


\section{Joints

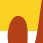

shoulder chondrolysis following arthroscopic surgery treated with total shoulder arthroplasty. J Shoulder Elbow Surg 2008; 17:380-388.

14. Giannini S, Buda R, Grigolo B, et al. Bipolar fresh osteochondral allograft of the ankle. Foot Ankle Int 2010; 31:38-46.

15. Czitrom AA, Keating S, Gross AE. The viability of articular cartilage in fresh osteochondral allografts after clinical transplantation. J Bone Joint Surg Am 1990; 72:574-581.

16. Giannini S, Buda R, Cavallo M, et al. Bipolar fresh osteochondral allograft for the treatment of glenohumeral posttraumatic arthritis. Knee Surg Sports Traumatol Arthrosc 2012; 20:1953-1957.

17. Armitage MS, Faber KJ, Drosdowech DS, et al. Humeral head bone defects: remplissage, allograft, and arthroplasty. Orthop Clin North Am 2010; 41:417-425.

18. Emmerson BC, Görtz S, Jamali AA, et al. Fresh osteochondral allografting in the treatment of osteochondritis dissecans of the femoral condyle. Am J Sports Med 2007; 35:907-914.

19. Raiss P, Aldinger PR, Kasten P, et al. Total shoulder replace- ment in young and middle-aged patients with glenohumeral osteoarthritis. J Bone Joint Surg Br 2008; 90:764-769.

20. Evans KN, Providence BC. Case report: fresh-stored osteochondral allograft for treatment of osteochondritis dissecans the femoral head. Clin Orthop Relat Res 2010; 468:613-618.

21. Weinstein DM, Bucchieri JS, Pollock RG, et al. Arthroscopic debridement of the shoulder for osteoarthritis. Arthroscopy 2000; 16:471-476.

22. Gross AE, Silverstein EA, Falk J, et al. The allotransplantation of partial joints in the treatment of osteoarthritis of the knee. Clin Orthop Relat Res 1975; 108:7-14.

23. Kim CW, Jamali A, Tontz W Jr, et al. Treatment of post-traumatic ankle arthrosis with bipolar tibiotalar osteochondral shell allografts. Foot Ankle Int 2002; 23:1091-1102.

24. McCulloch PC, Kang RW, Sobhy MH, et al. Prospective evaluation of prolonged fresh osteochondral allograft transplantation of the femoral condyle: minimum 2-year follow-up. Am J Sports Med 2007; 35:411-420. 